\section{Transtorno mental comum na população idosa: pesquisa de base populacional no Município de Campinas, São Paulo, Brasil}

\author{
Common mental disorders among elderly individuals: \\ a population-based study in Campinas, São Paulo \\ State, Brazil \\ Trastornos mentales más frecuentes entre las \\ personas de edad avanzada: un estudio basado \\ en la población en Campinas, São Paulo, Brasil
}

Flávia Silva Arbex Borim 1 Marilisa Berti de Azevedo Barros 1 Neury José Botega 1

\section{Resumo}

${ }_{1}^{1}$ Faculdade de Ciências Médicas, Universidade Estadual de Campinas, Campinas, Brasil.

Correspondência F. S. A. Borim Faculdade de Ciências Médicas, Universidade Estadual de Campinas. Rua Padre Francisco de Abreu Sampaio 269, apto. $32 B$ Campinas, $S P$ 13036-140, Brasil. flarbex@hotmail.com

\begin{abstract}
This study analyzed common mental disorders
Abstract

in the elderly according to demographic and socioeconomic characteristics, health-related behavior, and disease history. This was a crosssectional, population-based study with a cluster sample using data from a health survey conducted in Campinas, São Paulo State, Brazil, in 2008. SRQ-20 was used to evaluate common mental disorders. Adjusted prevalence ratios were estimated using multiple Poisson regression. Prevalence of common mental disorders was 29.7\%, and higher rates were associated with female gender, age 80 years or older, lower income, not working, sedentary lifestyle, poor self-rated health, and greater number of chronic diseases. Prevalence ratios were higher in the subscale of depressive thinking. The results provide direct backing for planning interventions focused on health of the elderly, with an emphasis on elderly that work and follow healthy lifestyles. The study highlights the need for attention by health professionals to depressive disorder in the elderly.
\end{abstract}

Mental Disorders; Mental Health; Aged
Analisou-se o transtorno mental comum em idosos segundo variáveis demográficas, socioeconômicas, de comportamentos relacionados à saúde e morbidades. Trata-se de estudo transversal de base populacional, com amostra por conglomerados. A pesquisa utilizou dados de inquérito de saúde realizado em Campinas, São Paulo, Brasil, em 2008. Foi usado o questionário SRQ-20 para avaliar o transtorno mental comum. Foram estimadas razões de prevalências ajustadas por meio de regressão múltipla de Poisson. A prevalência de transtorno mental comum foi $29,7 \%$ e significativamente mais elevada no sexo feminino, nos idosos com 80 anos ou mais, menor renda, que não trabalhavam, sedentários, que avaliaram sua saúde como ruim/muito ruim e com maior número de doenças crônicas. Maiores razões de prevalências foram detectadas na subescala de pensamentos depressivos. Os resultados trazem subsídios para o planejamento de intervenções voltadas à saúde dos idosos, com ênfase nos idosos que trabalham e com hábitos de vida saudáveis. Apontam a necessidade de atenção dos profissionais para o quadro depressivo na terceira idade.

Transtornos Mentais; Saúde Mental; Idoso 


\section{Introdução}

A população brasileira vem passando, nas últimas décadas, por um processo acelerado de transição demográfica, com aumento significativo do segmento de idosos. Essa mudança demográfica, pela repercussão que tem para a sociedade como um todo, especialmente no contexto de desigualdade social, pobreza e fragilidade das instituições, traz uma série de demandas e desafios para pesquisadores e gestores dos sistemas de saúde 1 . Em 2010, os idosos já representavam $7,4 \%$ da população brasileira, sendo o segmento de 80 anos e mais o que havia apresentado a maior taxa de crescimento (70\%) na década anterior 2 .

Com o aumento da expectativa de vida dos idosos, crescem as prevalências de doenças crônicas, os riscos de limitações físicas, de perdas cognitivas, de declínio sensorial e de propensão a acidentes e a isolamento social. Além dos aspectos físicos, a saúde mental também é comprometida, com consequente deterioração da saúde dos idosos. Os quadros psiquiátricos nos idosos incluem, destacadamente, a demência, estados depressivos, transtornos ansiosos e mesmo quadros psicóticos 3 , sendo, entretanto, a depressão o mais importante problema de saúde mental nessa faixa etária 4 .

Os sintomas psiquiátricos não psicóticos, incluídos no conjunto designado por transtorno mental comum, são caracterizados por sintomas como irritabilidade, fadiga, insônia, dificuldade de concentração, esquecimento, ansiedade e queixas somáticas ${ }^{5}$. Os pacientes apresentam problemas agudos de ansiedade e depressão, com sintomas menos graves, associados a eventos estressantes da vida, com predomínio de sintomas somáticos em relação aos sintomas psicológicos 6 .

Os transtornos mentais comuns são frequentes nos pacientes que demandam os serviços de saúde, mas ao manifestarem-se por meio de queixas somáticas inespecíficas, apresentam-se subestimados entre os diagnósticos referidos 7 . Se por um lado a Política Nacional de Saúde da Pessoa Idosa ${ }^{8}$ e o texto da I Conferência Nacional dos Direitos do Idoso afirmam que a saúde mental é questão que deve ser levada em consideração pela sua alta prevalência 9 , o subdiagnóstico dificulta o encaminhamento e o cuidado adequado dos transtornos mentais que acometem os idosos 7.

O Self Reporting Questionnaire 20 (SRQ-20) é um instrumento desenvolvido pela Organização Mundial da Saúde (OMS) para detecção de transtorno mental comum na população geral; ele foi submetido a um estudo de validação no Brasil, por Mari \& Williams 10 em 1986. Mais recente- mente, Scazufca et al. ${ }^{11}$ analisaram a validade do instrumento para a população idosa e sugeriram diferente ponto de corte para esse grupo.

Esse questionário tem sido amplamente utilizado em inquéritos de saúde de base populacional pelo fácil uso e custo reduzido 12 . Estudos desenvolvidos com o SRQ-20, em população de adultos incluindo idosos, apontaram que a prevalência de transtorno mental comum variou de $17 \%$ a $28,5 \%$ e encontraram associação positiva do mesmo com o sexo feminino, idade avançada, com menor renda e baixa escolaridade 7,13,14,15.

Poucos estudos brasileiros investigaram o transtorno mental comum especificamente em população idosa. Neste grupo etário, é mais comum encontrar estudos que utilizaram a Escala de Depressão Geriátrica (GDS) para avaliar sintomas depressivos 16,17 e que detectaram a relação positiva entre depressão e presença de incapacidades, diminuição da qualidade de vida, maior consumo de serviços de saúde e aumento da mortalidade 18. Entre os estudos brasileiros que utilizaram o SRQ-20 na avaliação de saúde mental de idosos, um analisou a validade do instrumento 11 e outro, com uma amostra de idosos moradores de São Paulo, avaliou a associação entre dimensões de religiosidade e prevalência de transtorno mental comum 19.

Identificar a prevalência do transtorno mental comum, os fatores associados e o impacto no estado geral de saúde dos idosos possibilita uma melhor compreensão dos profissionais de saúde e orientação no planejamento de intervenções.

Como a população idosa brasileira vem crescendo significativamente nas últimas décadas e a prevalência de transtorno mental comum bem como o seu impacto no estado geral de saúde dos idosos têm sido pouco estudados, os objetivos desta pesquisa foram: analisar a prevalência de transtorno mental comum, identificando os subgrupos demográficos e socioeconômicos de idosos mais susceptíveis ao transtorno; analisar a associação deste transtorno com comportamentos de saúde e morbidades; e identificar as dimensões do SRQ-20 mais comprometidas nos indivíduos com transtorno mental comum desse grupo etário.

\section{Material e métodos}

Este estudo é parte de uma pesquisa maior constituída pelo inquérito de saúde de base populacional realizado em 2008 no Município de Campinas, São Paulo (ISA-Camp 2008) que coletou informações de pessoas não institucionalizadas, residentes na área urbana do município. 


\section{Processo amostral do ISA-Camp 2008}

Foi utilizada amostragem por conglomerados, em dois estágios. No primeiro estágio, foram sorteados 50 setores censitários da área urbana do município com probabilidade proporcional ao tamanho, expresso pelo número de domicílios. Nesses 50 setores, foi feita pesquisa de campo para arrolamento dos domicílios particulares existentes. No segundo estágio, foi feito o sorteio de uma amostra de domicílios considerando que o total de entrevistas por setor não deveria ultrapassar 20 para cada domínio de idade (adolescentes, adultos e idosos).

Optou-se pelo sorteio de amostras de tamanhos iguais, de mil pessoas, para cada um dos grupos etários. Com esse tamanho de amostra seria possível estimar uma proporção de 0,50 , que corresponde à máxima variabilidade para a frequência dos eventos estudados, com erro de amostragem entre 4 e 5 pontos percentuais, com nível de $95 \%$ de confiança e tendo em conta um efeito de delineamento igual a 2.

Prevendo-se $20 \%$ de recusas e de domicílios vagos, foram sorteados 2.150, 700 e 3.900 respectivos aos três grupos de idade. Dessa forma, para a obtenção de ao menos mil entrevistas de idosos, foram sorteados 3.900 domicílios em que deveriam ser entrevistados todos os idosos que residissem no domicílio sorteado (detalhes sobre o processo amostral encontram-se disponíveis em http://www.fcm.unicamp.br/centros/ccas/ arquivos/plano_de_amostragem.pdf, acessado em 25/Mar/2013).

\section{Instrumento e coleta de dados}

As informações foram coletadas por meio de questionário aplicado por entrevistadores treinados e respondidos diretamente pelos idosos sorteados, a não ser nos casos que eles se encontravam impossibilitados, um cuidador ou parente responsável respondia por ele. Os conjuntos de variáveis analisadas no presente estudo foram:

- Transtorno mental comum: avaliado pelo (SRQ 20): o questionário é composto por 20 questões sobre sintomas físicos e psíquicos, com respostas dicotômicas. O ponto de corte utilizado foi maior ou igual a 5 para os possíveis casos de transtorno mental comum, sendo o mesmo ponto de corte para ambos os gêneros 11 .

- Características demográficas: sexo, idade e estado conjugal (casado, viúvo, separado ou solteiro). - Características socioeconômicas: escolaridade (em anos de estudo), ocupação e renda familiar mensal per capita (em salários mínimos, cujos valores foram de $\mathrm{R} \$ 415,00$ de janeiro a abril $/ 2008$ e de $\mathrm{R} \$ 450,00$ de maio/2008 a abril/2009).
- Comportamentos relacionados à saúde, compreendendo: (a) atividade física em contexto de lazer: foram classificados como ativos os idosos que praticavam ao menos 150 minutos por semana, distribuídos no mínimo por 3 dias; como insuficientemente ativos os que praticavam menos que 150 minutos ou mais de 150 minutos, mas em menos de 3 dias na semana, e como sedentários os que não praticavam nenhum tipo de atividade física de lazer em nenhum dia da semana; (b) uso abusivo de bebidas alcoólicas, avaliado pelo Alcohol Use Disorder Identification Test (AUDIT) 20 que é composto por dez questões e identifica o risco de abuso/dependência de álcool quando seu escore (faixa de 0-40) é igual a oito ou mais ${ }^{21}$. Foi também avaliada a frequência do consumo de bebida alcoólica categorizada em: não consome, consome 1-4 vezes/mês, 2-3 vezes/semana e 4 ou mais vezes/semana; (c) tabagismo: fumante, ex-fumante e nunca fumou. Foram considerados como ex-fumantes as pessoas que referiram ter fumado ao menos 100 cigarros na vida e haviam cessado com o consumo; foram classificados como fumantes aqueles que persistiam com o consumo do tabaco por ocasião de entrevista; e (d) frequência semanal do consumo de frutas e verduras-hortaliças categorizada em $\geq$ 4 vezes/semana e $<4$ vezes/semana.

- Estado de saúde e morbidades, compreendendo: (a) índice de massa corporal (IMC): foi calculada com base em informação autorreferida de peso e altura. Foram usados os pontos de corte adequados para idosos que levam em consideração as modificações na composição corporal que ocorrem com o envelhecimento 22. Os idosos foram categorizados em: baixo peso/ eutrófico (IMC $\leq 27 \mathrm{~kg} / \mathrm{m}^{2}$ ), sobrepeso (IMC > 27 $\mathrm{a}<30 \mathrm{~kg} / \mathrm{m}^{2}$ ) e obeso (IMC $\geq 30 \mathrm{~kg} / \mathrm{m}^{2}$ ); (b) autoavaliação da saúde obtida por meio da pergunta: "Em geral, você diria que sua saúde é: excelente, muito boa, boa, ruim ou muito ruim?"; e (c) morbidades: hipertensão, diabetes, doença do coração, tumor/câncer, reumatismo/artrite/artrose, osteoporose, asma/bronquite/enfisema, tendinite/lesão por esforço repetido (LER)/distúrbio osteomuscular relacionado ao trabalho (DORT) e problemas de circulação; número de morbidades crônicas referidas entre as acima relacionadas.

\section{Análise de dados}

Os dados do inquérito foram digitados em banco desenvolvido com o uso do software Epi Data, versão 3.1 (Epidata Association, Odense, Dinamarca) e submetidos à avaliação de consistência. Para as análises deste estudo, foram produzidas estimativas de prevalências e respec- 
tivos intervalos de 95\% de confiança (IC95\%). As associações entre variáveis independentes e o transtorno mental comum foram analisadas pelo teste qui-quadrado. Também foram usadas as análises de regressão simples e múltipla de Poisson para estimar razões de prevalência (RP) brutas e ajustadas. Foi desenvolvido modelo de regressão múltipla de Poisson em três etapas. Na primeira etapa, foram introduzidas as variáveis demográficas e socioeconômicas que apresentaram um nível de significância menor de 20\% ( $p<0,20)$ na associação com a variável dependente. Permaneceram no modelo aquelas com $\mathrm{p}<0,05$. Na segunda etapa, além das variáveis que permaneceram na etapa anterior, foram acrescidas as variáveis de comportamentos relacionados à saúde e se mantiveram aquelas com $\mathrm{p}<0,05$. Na terceira etapa, foram incluídas as variáveis IMC, autoavaliação de saúde e número de morbidades crônicas referidas, mantendo-se nessa etapa aquelas com $\mathrm{p}<0,05$. A análise dos dados foi realizada com os comandos svy do software Stata versão 11.0 (Stata Corp., College Station, Estados Unidos), utilizando-se as ponderações decorrentes do desenho amostral e considerando a existência das unidades primárias de amostragem (UPA).

\section{Procedimentos éticos}

O presente estudo foi aprovado pelo Comitê de Ética em Pesquisa da Faculdade de Ciências Médicas da Universidade Estadual de Campinas, em adendo ao parecer no 079/2007 que havia aprovado o projeto do ISA-Camp 2008. Todos os participantes assinaram Termo de Consentimento Livre e Esclarecido.

\section{Resultados}

Dos domicílios que haviam sido sorteados para as entrevistas com idosos, houve uma perda de $6,5 \%$ por impossibilidade de encontrar um morador ou devido à recusa dele em arrolar os indivíduos que moravam no domicílio. Dos 1.558 idosos identificados nos domicílios sorteados, $2,4 \%$ recusaram-se a participar da pesquisa, sendo entrevistados 1.520 idosos. Dentre esses, em $5,8 \%$ dos casos, as entrevistas foram feitas com um cuidador ou um familiar e foram excluídas do presente estudo.

Assim, foram analisados os dados de 1.432 idosos com idade média de 69,9 anos, sendo $57,2 \%$ do sexo feminino. Na Tabela 1 , observa-se que $57,6 \%$ dos indivíduos eram casados; $34,4 \%$ tinham menos de quatro anos de estudo; $60 \%$ tinham renda per capita maior que um salário mínimo e 49,5\% eram aposentados que já não trabalhavam, enquanto $21,2 \%$ continuavam trabalhando sendo ou não aposentados.

A prevalência de transtorno mental comum foi de 29,7\% (IC95\%: 25,5-34,2). Observou-se que os idosos do sexo feminino, de 70 anos ou mais, com escolaridade $<4$ anos, com renda per capita menor que 0,5 salário mínimo e os que não trabalhavam apresentaram maior prevalência de transtorno mental comum (Tabela 1).

Os indivíduos que eram ativos ou insuficientemente ativos no lazer, que consumiam bebida alcoólica com frequência de até 3 vezes por semana e os que consumiam frutas e verduras 4 ou mais vezes por semana apresentaram prevalência significativamente menor de transtorno mental comum em relação às respectivas categorias de referência (Tabela 2 ).

Na Tabela 3, é apresentada a prevalência do transtorno mental comum segundo estado de saúde e morbidades. Os obesos apresentaram uma prevalência maior de transtorno mental comum quando comparados aos indivíduos eutróficos ou de baixo peso. Verificou-se aumento da prevalência com a piora da autoavaliação de saúde e com o aumento do número de morbidades referidas. As prevalências de transtorno mental comum foram significativamente mais elevadas na presença de todas as doenças pesquisadas: hipertensão, diabetes, doença do coração, reumatismo/artrite/artrose, osteoporose, problema de circulação e tendinite (LER/DORT), com exceção de asma/enfisema e câncer. As doenças que apresentaram associações mais fortes com transtorno mental comum foram reumatismo/artrite/artrose com razão de prevalência $\mathrm{RP}=1,86$ (IC95\%: 1,50-2,32) e tendinite com $\mathrm{RP}=1,91$ (IC95\%: 1,53-2,38) (dados não apresentados em tabela).

Com o uso do modelo hierárquico de regressão múltipla de Poisson, verificou-se maior prevalência de transtorno mental comum nas mulheres, nos indivíduos com 80 anos ou mais, com renda per capita menor que 0,5 salário mínimo, nos aposentados, nas donas de casa e nos desempregados. Os indivíduos ativos ou insuficientemente ativos no lazer e aqueles que consumiam bebida alcoólica 2 a 3 vezes por semana apresentaram menor prevalência de transtorno mental comum, com RP de 0,72 (IC95\%: 0,570,90) e 0,39 (IC95\%: 0,20-0,75), respectivamente (Tabela 4).

Prevalências mais elevadas de transtorno mental comum foram encontradas à medida da piora na autoavaliação de saúde. Indivíduos que avaliaram a própria saúde como ruim/muito ruim apresentaram uma $\mathrm{RP}$ de 3,46 quando comparadas aos idosos com autoavaliação de saúde 
Prevalência e razão de prevalência (RP) de transtorno mental comum segundo variáveis demográficas e socioeconômicas em idosos. Inquérito de Saúde de Campinas (ISA-Camp), 2008.

\begin{tabular}{|c|c|c|c|c|c|}
\hline Variáveis & $\mathrm{n}$ & $\%$ & Prevalência & RP bruta (IC95\%) & RP Ajustada * (IC95\%) \\
\hline Sexo & & & $p<0,0001$ & & \\
\hline Masculino & 580 & 42,8 & 19,4 & 1,00 & 1,00 \\
\hline Feminino & 852 & 57,2 & 37,4 & $1,93(1,65-2,26)$ & $1,88(1,59-2,22)$ \\
\hline Total & 1.432 & 100,0 & 29,7 & & \\
\hline Faixa etária (anos) & & & $p=0,0001$ & & \\
\hline $60-69$ & 800 & 55,9 & 24,3 & 1,00 & 1,00 \\
\hline $70-79$ & 470 & 32,8 & 32,6 & $1,34(1,11-1,61)$ & $1,31(1,09-1,58)$ \\
\hline 80 e mais & 162 & 11,3 & 47,6 & $1,95(1,44-2,65)$ & $1,86(1,39-2,49)$ \\
\hline Estado conjugal & & & $p<0,0001$ & & \\
\hline Casado & 813 & 57,6 & 24,6 & 1,00 & 1,00 \\
\hline Viúvo & 416 & 28,2 & 40,6 & $1,65(1,33-2,04)$ & $1,15(0,90-1,47)$ \\
\hline Separado & 113 & 7,9 & 27,1 & $1,10(0,79-1,52)$ & $1,01(0,72-1,41)$ \\
\hline Solteiro & 90 & 6,3 & 30,1 & $1,22(0,87-1,71)$ & $0,98(0,68-1,42)$ \\
\hline Escolaridade do indivíduo (em anos) & & & $p=0,0022$ & & \\
\hline$\geq 8$ & 432 & 31,6 & 22,0 & 1,00 & 1,00 \\
\hline $4-7$ & 493 & 34,0 & 30,1 & $1,37(1,01-1,85)$ & $1,21(0,91-1,60)$ \\
\hline$<4$ & 504 & 34,4 & 36,3 & $1,64(1,28-2,11)$ & $1,35(1,07-1,71)$ \\
\hline Renda per capita (salário mínimo) & & & $p=0,0025$ & & \\
\hline$>1,0$ & 848 & 60,0 & 26,2 & 1,00 & 1,00 \\
\hline $0,5-1,0$ & 359 & 24,5 & 33,1 & $1,26(1,03-1,54)$ & $1,18(0,97-1,43)$ \\
\hline$<0,5$ & 225 & 15,5 & 37,7 & $1,44(1,18-1,75)$ & $1,37(1,11-1,69)$ \\
\hline Ocupação & & & $p<0,0001$ & & \\
\hline Trabalho/Aposentado + trabalho & 295 & 21,2 & 12,2 & 1,00 & 1,00 \\
\hline Aposentado & 702 & 49,5 & 30,8 & $2,51(1,76-3,58)$ & $2,10(1,46-3,02)$ \\
\hline Dona de casa & 390 & 26,2 & 41,2 & $3,36(2,35-4,79)$ & $2,16(1,48-3,16)$ \\
\hline Desempregado/outros & 44 & 3,1 & 35,9 & $2,92(1,62-5,26)$ & $3,05(1,73-5,38)$ \\
\hline
\end{tabular}

IC95\%: intervalo de $95 \%$ confiança.

* Ajustada por sexo e idade.

excelente ou muito boa. Apresentar uma morbidade ou mais esteve associada positivamente com o transtorno mental comum (Tabela 4).

$\mathrm{Na}$ análise dos itens específicos do instrumento SRQ-20, detectou-se que os que mais diferenciavam os idosos com transtorno mental comum dos demais foram: sentir-se uma pessoa inútil, sem préstimo ( $R P=25,02$; IC95\%: 12,95-48,35), sentir-se incapaz de desempenhar um papel útil na sua vida $(\mathrm{RP}=17,31$; IC95\%: 9,65-31,07), ter perdido o interesse pelas coisas (RP = 16,22; IC95\%: 10,02-26,25), ter tido a idéia de acabar com a vida (RP = 15,57; IC95\%: 4,11$58,95)$, ter chorado mais que de costume $(\mathrm{RP}=$ 15,1; IC95\%: 9,19-24,9) e sentir-se cansado o tempo todo $(\mathrm{RP}=12,59$; IC95\%: 8,59-18,45). As maiores RP foram encontradas na subescala de pensamentos depressivos (Tabela 5).

\section{Discussão}

A prevalência de transtorno mental comum detectada para a população idosa de Campinas foi similar à encontrada em outros estudos brasileiros de base populacional que analisaram população adulta que incluía os idosos 13,23. Costa et al. 13, em 2002, observaram uma presença de transtorno mental comum em $28,5 \%$ dos indivíduos com idade acima de 40 anos, utilizando ponto de corte de 6 e 7 respostas positivas para homens e mulheres, respectivamente. A prevalência de transtorno mental comum entre as mulheres foi maior que a dos homens, o que é consistente com os achados da literatura 15,24.

Com o aumento da idade, constatou-se maior prevalência do transtorno mental comum, e os idosos de 80 anos ou mais apresentaram 
Tabela 2

Prevalência e razão de prevalência (RP) de transtorno mental comum segundo comportamentos relacionados à saúde. Inquérito de Saúde de Campinas (ISA-Camp), 2008.

\begin{tabular}{|c|c|c|c|c|}
\hline Variáveis & $\mathrm{n}$ & Prevalência & RP bruta (IC95\%) & RP ajustada * (IC95\%) \\
\hline Tabagismo & & $p=0,6382$ & & \\
\hline Nunca fumou & 973 & 30,5 & 1,00 & 1,00 \\
\hline Ex-fumante & 292 & 26,7 & $0,93(0,72-1,19)$ & $1,18(0,92-1,52)$ \\
\hline Fumante & 166 & 28,4 & $0,87(0,63-1,21)$ & $1,10(0,82-1,48)$ \\
\hline Audit & & $p=0,9968$ & & \\
\hline Negativo & 1.372 & 29,7 & 1,00 & 1,00 \\
\hline Positivo & 59 & 29,7 & $1,00(0,57-1,73)$ & $1,64(0,98-2,76)$ \\
\hline Ingestão de álcool & & $p<0,0001$ & & \\
\hline Não bebe & 991 & 34,2 & 1,00 & 1,00 \\
\hline $1-4$ vezes/mês & 299 & 22,6 & $0,66(0,51-0,86)$ & $0,74(0,58-0,96)$ \\
\hline 2-3 vezes/semana & 73 & 8,9 & $0,26(0,13-0,50)$ & $0,33(0,17-0,65)$ \\
\hline$\geq 4$ vezes/semana & 68 & 20,3 & $0,59(0,35-0,99)$ & $0,90(0,53-1,54)$ \\
\hline Atividade física de lazer & & $p=0,0002$ & & \\
\hline Sedentário & 965 & 33,8 & 1,00 & 1,00 \\
\hline Insuficientemente ativo & 156 & 22,4 & $0,66(0,47-0,93)$ & $0,67(0,50-0,91)$ \\
\hline Ativo & 311 & 20,8 & $0,61(0,46-0,81)$ & $0,67(0,51-0,87)$ \\
\hline Consumo de frutas/verduras (frequência por semana) & & $p=0,0510$ & & \\
\hline$<4$ vezes & 534 & 32,9 & 1,00 & 1,00 \\
\hline$\geq 4$ vezes & 898 & 27,7 & $0,84(0,71-1,00)$ & $0,77(0,66-0,92)$ \\
\hline
\end{tabular}

Audit: Alcohol Use Disorder Identification Test; IC95\%: intervalo de 95\% confiança.

* Ajustada por sexo e idade.

\section{Tabela 3}

Prevalência e razão de prevalência (RP) de transtorno mental comum segundo índice de massa corporal (IMC), autoavaliação de saúde e morbidades. Inquérito de Saúde de Campinas (ISA-Camp), 2008

\begin{tabular}{|c|c|c|c|c|}
\hline Variáveis & $\mathbf{n}$ & Prevalência & RP bruta (IC95\%) & RP ajustada * (IC95\%) \\
\hline IMC & & $p=0,0232$ & & \\
\hline Baixo peso/Eutrófico & 910 & 26,7 & 1,00 & 1,00 \\
\hline Sobrepeso & 265 & 31,1 & $1,16(0,91-1,47)$ & $1,17(0,93-1,47)$ \\
\hline Obeso & 208 & 37,3 & $1,39(1,09-1,77)$ & $1,33(1,06-1,67)$ \\
\hline Autoavaliação de saúde & & $p<0,0001$ & & \\
\hline Excelente & 116 & 11,7 & 1,00 & 1,00 \\
\hline Muito boa & 231 & 13,5 & $1,15(0,58-2,24)$ & $1,18(0,60-2,32)$ \\
\hline Boa & 927 & 29,4 & $2,51(1,41-4,46)$ & $2,42(1,36-4,31)$ \\
\hline Ruim & 144 & 68,8 & $5,87(3,47-9,94)$ & $5,95(3,58-9,89)$ \\
\hline Muito ruim & 14 & 78,9 & $6,74(3,62-12,54)$ & $6,66(3,29-13,47)$ \\
\hline Número de doenças & & $p<0,0001$ & & \\
\hline 0 & 278 & 12,0 & 1,00 & 1,00 \\
\hline $1-2$ & 667 & 25,3 & $2,12(1,37-3,27)$ & $1,96(1,28-3,01)$ \\
\hline $3-4$ & 353 & 39,8 & $3,33(2,06-5,37)$ & $2,78(1,71-4,53)$ \\
\hline 5 ou mais & 133 & 64,2 & $5,38(3,32-8,70)$ & $4,45(2,77-7,15)$ \\
\hline
\end{tabular}

IC95\%: intervalo de $95 \%$ de confiança.

* Ajustada por sexo e idade. 
Tabela 4

Modelo hierárquico com regressão de Poisson. Inquérito de Saúde de Campinas (ISA-Camp), 2008.

\begin{tabular}{|c|c|c|c|c|c|c|c|c|c|}
\hline \multirow[t]{2}{*}{ Variáveis } & \multicolumn{3}{|c|}{ Primeira etapa * } & \multicolumn{3}{|c|}{ Segunda etapa ** } & \multicolumn{3}{|c|}{ Terceira etapa } \\
\hline & RP & IC95\% & Valor de $p$ & RP & IC $95 \%$ & Valor de $p$ & RP & IC95\% & Valor de $p$ \\
\hline \multicolumn{10}{|l|}{ Sexo } \\
\hline Masculino & 1,00 & & & 1,00 & & & 1,00 & & \\
\hline Feminino & 1,69 & $1,40-2,05$ & 0,000 & 1,58 & $1,30-1,91$ & 0,000 & 1,45 & $1,21-1,73$ & 0,000 \\
\hline \multicolumn{10}{|l|}{ Faixa etária (anos) } \\
\hline $60-69$ & 1,00 & & & 1,00 & & & 1,00 & & \\
\hline $70-79$ & 1,18 & $0,99-1,40$ & 0,058 & 1,16 & $0,98-1,37$ & 0,081 & 1,06 & $0,91-1,23$ & 0,453 \\
\hline 80 e mais & 1,63 & $1,24-2,14$ & 0,001 & 1,59 & $1,21-2,08$ & 0,001 & 1,68 & $1,32-2,15$ & 0,000 \\
\hline \multicolumn{10}{|l|}{ Ocupação } \\
\hline $\begin{array}{l}\text { Trabalho/Aposentado + } \\
\text { trabalho }\end{array}$ & 1,00 & & & 1,00 & & & 1,00 & & \\
\hline Aposentado & 2,07 & $1,44-2,98$ & 0,000 & 2,00 & $1,39-2,87$ & 0,000 & 1,66 & $1,16-2,37$ & 0,006 \\
\hline Dona de casa & 2,10 & $1,44-3,07$ & 0,000 & 1,98 & $1,36-2,88$ & 0,001 & 1,75 & $1,19-2,58$ & 0,005 \\
\hline Desempregado/Outros & 2,77 & $1,59-4,85$ & 0,001 & 2,44 & $1,41-4,21$ & 0,002 & 1,57 & $0,94-2,61$ & 0,080 \\
\hline \multicolumn{10}{|l|}{ Renda per capita (salário mínimo) } \\
\hline$>1$ & 1,00 & & & 1,00 & & & 1,00 & & \\
\hline $0,5-1$ & 1,14 & $0,94-1,39$ & 0,176 & 1,08 & $0,89-1,32$ & 0,413 & 1,03 & $0,86-1,23$ & 0,720 \\
\hline$<0,5$ & 1,28 & $1,06-1,55$ & 0,010 & 1,22 & $1,01-1,48$ & 0,034 & 1,12 & $0,94-1,33$ & 0,180 \\
\hline \multicolumn{10}{|l|}{ Atividade física de lazer } \\
\hline Sedentário & & & & 1,00 & & & 1,00 & & \\
\hline Ativo + Insuficientemente ativo & & & & 0,72 & $0,57-0,90$ & 0,005 & 0,80 & $0,66-0,98$ & 0,033 \\
\hline \multicolumn{10}{|l|}{ Ingestão de álcool } \\
\hline Não bebe & & & & 1,00 & & & 1,00 & & \\
\hline 1-4 vezes/mês & & & & 0,82 & $0,64-1,04$ & 0,112 & 0,92 & $0,72-1,17$ & 0,490 \\
\hline $2-3$ vezes/semana & & & & 0,39 & $0,20-0,75$ & 0,006 & 0,44 & $0,23-0,85$ & 0,016 \\
\hline$\geq 4$ vezes/semana & & & & 0,93 & $0,57-1,53$ & 0,782 & 1,12 & $0,72-1,75$ & 0,600 \\
\hline \multicolumn{10}{|l|}{ Autoavaliação de saúde } \\
\hline Excelente/Muito boa & & & & & & & 1,00 & & \\
\hline Boa & & & & & & & 1,76 & $1,27-2,44$ & 0,001 \\
\hline Muito ruim/Ruim & & & & & & & 3,46 & $2,44-4,91$ & 0,000 \\
\hline \multicolumn{10}{|l|}{ Morbidade } \\
\hline 0 & & & & & & & 1,00 & & \\
\hline $1-2$ & & & & & & & 1,55 & $1,05-2,27$ & 0,027 \\
\hline $3-4$ & & & & & & & 1,99 & $1,27-3,13$ & 0,004 \\
\hline 5 ou mais & & & & & & & 2,78 & $1,77-4,36$ & 0,000 \\
\hline
\end{tabular}

IC95\%: intervalo de 95\% de confiança; RP: razão de prevalência.

* RP ajustadas pelas variáveis demográficas e socioeconômicas;

** RP ajustadas por todas as variáveis da tabela.

prevalência 2,86 vezes maior que os de 60-69 anos. Outros estudos apontam um gradiente crescente de transtorno mental comum com o aumento da idade 7,25. Muitos fatores podem contribuir para tal aumento, como o crescimento do número de morbidades e incapacidades, eventos estressantes de vida, isolamento social e dificuldades econômicas 7 .

A associação entre o nível socioeconômico e transtorno mental comum tem sido observa- da na literatura com adultos e idosos 26,27. Um estudo transversal, com amostra de 562 idosos residentes no Município de Feira de Santana, Bahia, Brasil, observou um aumento de transtorno mental comum no estrato de baixa renda 27 . A maior prevalência de transtorno mental comum em pessoas com pior nível socioeconômico pode estar relacionada a condições inadequadas de vida, à pior qualidade de moradia e transporte, à maior dificuldade de acesso a cuidados médicos, 
Prevalência e razão de prevalência (RP) dos problemas, nos últimos 30 dias, relacionados às questões do Self-Reporting Questionnaire (SRQ-20), segundo presença ou não de transtorno mental comum nos idosos. Inquérito de Saúde de Campinas (ISA-Camp), 2008.

\begin{tabular}{|c|c|c|c|c|}
\hline Perguntas & Normal (\%) & Transtorno mental comum (\%) & Total (\%) & $\operatorname{RP}(\mathrm{IC} 95 \%)$ * \\
\hline \multicolumn{5}{|l|}{ Humor depressivo-ansioso } \\
\hline Assusta-se com facilidade? & 11,2 & 45,7 & 21,5 & $4,08(3,10-5,36)$ \\
\hline Sente-se nervoso(a), tenso(a) ou preocupado(a)? & 34,7 & 80,2 & 48,1 & $2,31(1,94-2,76)$ \\
\hline Tem se sentido triste ultimamente? & 9,1 & 64,9 & 25,7 & $7,09(5,54-9,09)$ \\
\hline Tem chorado mais do que de costume? & 2,2 & 33,2 & 11,4 & $15,1(9,19-24,9)$ \\
\hline \multicolumn{5}{|l|}{ Sintomas somáticos } \\
\hline Tem dores de cabeça frequentes? & 12,2 & 33,1 & 18,4 & $2,70(2,15-3,38)$ \\
\hline Tem falta de apetite? & 4,1 & 28,2 & 11,2 & $6,85(4,57-10,25)$ \\
\hline Dorme mal? & 15,9 & 55,2 & 27,6 & $3,46(2,71-4,43)$ \\
\hline Tem tremores nas mãos? & 5,2 & 27,8 & 11,9 & $5,33(3,90-7,30)$ \\
\hline Tem má digestão? & 8,4 & 36,9 & 16,9 & $4,36(3,23-5,88)$ \\
\hline Tem sensações desagradáveis no estômago? & 5,9 & 44,4 & 17,3 & $7,54(5,34-10,64)$ \\
\hline \multicolumn{5}{|l|}{ Decréscimo de energia vital } \\
\hline Tem dificuldade de pensar com clareza? & 5,0 & 40,9 & 15,7 & $8,13(5,44-12,16)$ \\
\hline $\begin{array}{l}\text { Encontra dificuldade para realizar com satisfação suas } \\
\text { atividades diárias? }\end{array}$ & 4,1 & 45,8 & 16,5 & $10,96(7,58-15,85)$ \\
\hline Tem dificuldade para tomar decisões? & 4,0 & 39,2 & 14,5 & $9,68(6,60-14,20)$ \\
\hline $\begin{array}{l}\text { Tem dificuldade no serviço, seu trabalho é penoso, causa } \\
\text { sofrimento? }\end{array}$ & 2,9 & 30,8 & 11,2 & $10,45(4,93-22,11)$ \\
\hline Sente-se cansado(a) o tempo todo? & 3,7 & 46,4 & 16,4 & $12,59(8,59-18,45)$ \\
\hline Você se cansa com facilidade? & 11,6 & 67,0 & 28,1 & $5,75(4,68-7,07)$ \\
\hline \multicolumn{5}{|l|}{ Pensamentos depressivos } \\
\hline $\begin{array}{l}\text { Sente-se incapaz de desempenhar um papel útil } \\
\text { na sua vida? }\end{array}$ & 1,8 & 32,4 & 10,9 & $17,31(9,65-31,07)$ \\
\hline Tem perdido o interesse pelas coisas? & 2,6 & 42,2 & 14,4 & $16,22(10,02-26,25)$ \\
\hline Você se sente uma pessoa inútil, sem préstimo? & 1,1 & 28,1 & 9,1 & $25,02(12,95-48,35)$ \\
\hline Tem tido a idéia de acabar com a vida? & 0,4 & 6,4 & 2,2 & $15,57(4,11-58,95)$ \\
\hline
\end{tabular}

IC95\%: intervalo de 95\% confiança.

à maior prevalência de morbidades e de estresse psicossocial 28 resultantes do menor acesso a oportunidades sociais ao longo da vida.

No presente estudo, os indivíduos que não trabalhavam apresentaram maior risco de transtorno mental comum. O trabalho abrange a aplicação das forças e faculdades humanas para alcançar determinado fim, além das atividades física e intelectual necessárias a qualquer tarefa, serviço ou empreendimento. Portanto, para o idoso, a inserção no trabalho representa a preservação da capacidade da saúde física e mental, da participação social e a valorização desses indivíduos como cidadãos ativos na sociedade 29,30. Com isso, verifica-se maior risco de transtorno mental comum nos indivíduos aposentados, donas de casa e desempregados em relação àqueles que trabalham. Tal achado sinaliza que aqueles que se mantêm no mercado apresentam maior preservação da saúde mental.

Quando observada a associação entre comportamentos relacionados à saúde e transtorno mental comum, a prática de atividade física no lazer apresentou uma associação inversa com o transtorno. Um estilo de vida ativo ou mesmo insuficientemente ativo pode controlar e evitar alguns sintomas de doenças, melhorar ou manter a aptidão física e melhorar a capacidade de realizar atividades diárias, proporcionando benefícios fisiológicos, psicológicos e sociais, que podem reduzir a ansiedade e melhorar o humor 27 . A prática da atividade física pode proporcionar uma relativa manutenção da autonomia e independência, habilidades fundamentais para o bem-estar do indivíduo. Especificamente a atividade física no lazer se destaca, entre os outros 
domínios da atividade física, por propiciar oportunidade de estabelecimento de contatos e de formação de redes sociais, que são fundamentais nas relações psicossociais e reequilíbrio emocional para essa faixa etária, exercendo um impacto significativo na saúde mental 27. Outros estudos também têm verificado associação positiva entre transtorno mental comum e sedentarismo no lazer em idosos 27,31 .

Quanto à ingestão do consumo de bebida alcoólica, os idosos que ingeriam de 2 a 3 vezes por semana apresentaram prevalência de transtorno mental comum significativamente inferior aos abstêmios. O consumo moderado de álcool pode ter um impacto psicológico positivo, com alívio do sofrimento emocional e ser eficaz para lidar com situações sociais e de ansiedade ${ }^{32}$. É importante enfatizar que as pessoas que envelhecem apresentam maior prevalência de doenças e consomem mais medicamentos o que tende a diminuir ou cessar a ingestão do consumo de álcool 33,34, mas no presente estudo, mesmo ajustado para número de morbidades referidas os transtornos mentais comuns, mostraram-se menos prevalentes nos idosos que consomem bebidas alcoólicas. Outros estudos de corte transversal observaram maior prevalência de ativos no lazer 35 , melhor qualidade de vida $36 \mathrm{e}$ melhor autoavaliação de saúde 37 nos idosos que consumiam bebidas alcoólicas moderadamente. No entanto, a associação do consumo moderado de álcool com variáveis relacionadas à saúde é geralmente resultante das variáveis de confusão e de interação complexa, difíceis de serem controladas 32, 38. É importante ressaltar que o consumo abusivo de álcool compromete a saúde dos idosos, sobretudo por serem mais susceptíveis ao risco de morbidades, fraturas e alterações cognitivas 39 .

A prevalência de transtorno mental comum apresentou um aumento significativo à medida que piora a autoavaliação da saúde, como também verificados por outros estudos brasileiros 40 , com a existência de gradiente crescente da RP com a piora da saúde percebida ${ }^{41}$. A autoavaliação de saúde é uma medida de julgamento subjetivo que cada pessoa faz sobre a qualidade de sua saúde física e mental e, assim, justifica-se a relação entre transtorno mental comum e autoavaliação de saúde. Entretanto, existem autores que verificaram maior influência do componente físico sobre a autoavaliação de saúde 42 .

A prevalência de transtorno mental comum apresentou um gradiente crescente com o aumento do número de morbidades referidas, atingindo uma RP de 2,78 no segmento com 5 ou mais morbidades. Outros autores têm observado esses gradientes e verificado que a prevalência de transtorno mental comum é cerca de 4 vezes maior de idosos com 4 a 5 morbidades relativamente aos que não apresentavam doenças crônicas 43,44 . As doenças crônicas, frequentemente encontradas nos idosos, associam-se à incapacidade funcional 45,46 , a aumento da vulnerabilidade e da dependência, contribuindo para o isolamento social e podendo, por conseguinte, aumentar a presença de sintomas somáticos, depressivos e ansiosos, entre outros 47 .

A subescala de pensamentos depressivos apresentou maiores RP nos idosos com transtorno mental comum em relação aos que não apresentavam o transtorno. A presença de sintomatologia depressiva está associada a uma série de comprometimentos da saúde do indivíduo, com piora na qualidade de vida e maior utilização dos recursos de saúde ${ }^{48}$. Esses achados podem ajudar a preparar os serviços de saúde para acolher, avaliar e tomar as medidas preventivas e curativas pertinentes ao grupo 49 .

Os diferentes pontos de corte utilizados para o SRQ-20 acarretam falta de comparação entre os estudos, mas é importante destacar que o instrumento foi traduzido em vários idiomas, suas características têm boa aceitação em populações com alto índice de analfabetismo e possibilita o uso em estudos epidemiológicos com indivíduos de todas as idades 11. Dentre as limitações do presente estudo, cabe destacar que o corte transversal não possibilita afirmar se o fator associado é determinante ou determinado pelo transtorno mental comum, como por exemplo, se as doenças crônicas antecederam ou sucederam o transtorno mental comum.

A prevalência do transtorno mental comum é elevada na população idosa estudada e os achados deste estudo constataram as maiores prevalências nos mais velhos e nos indivíduos que não trabalhavam. Conforme mencionado anteriormente, o segmento de 80 anos e mais é o grupo etário que mais cresce no país e é fundamental investir na autonomia e na vida saudável desses indivíduos, assim como prover atenção adequada às suas necessidades; outro foco deve-se dar ao trabalho na velhice, que representa uma autonomia e inserção do indivíduo na sociedade, preparar o idoso para o não trabalho e proporcionar a reinserção em novos grupos sociais, como por exemplo, estimular a procura de atividades de lazer, pode contribuir para uma melhor promoção da saúde na velhice.

Os resultados também trazem subsídios que possibilitam melhor orientar o planejamento de intervenções voltadas à saúde dos idosos, com ênfase nos hábitos de vida saudáveis, em especial na prática de atividade física, além de destacar a necessidade dos profissionais de saúde em 
identificar o transtorno mental comum nesse grupo etário, para melhor desenvolver estratégias e práticas para o cuidado de tais indivíduos. Apontam ainda que a identificação de transtorno mental comum em idosos associa-se mais for- temente aos itens e escala de pensamentos depressivos e, portanto, sugere-se maior atenção dos profissionais para o quadro depressivo na terceira idade.

\section{Resumen}

Se analizaron los trastornos mentales comunes en ancianos, de acuerdo con los comportamientos demográficos, socioeconómicos, de salud y morbilidad. Se realizó un estudio transversal, basado en la población de muestreo por conglomerados de los datos de una encuesta de salud realizada en Campinas, São Paulo, Brasil, en 2008. Se utilizó el SRQ-20 para evaluar la trastornos mentales comunes. Calculamos razones de prevalencia ajustadas por regresión de Poisson múltiple. La prevalencia de trastornos mentales comunes fue de $29,7 \%$, significativamente mayor en las mujeres, las personas mayores de 80 años, de bajos ingresos, que no trabajan, sedentarias, que calificaron su salud como mala/muy mala y con más enfermedades crónicas. Mayores tasas de prevalencia fueron encontradas en las subescalas de trastornos depresivos. Proporcionan información para la planificación de intervenciones centradas en la salud de las personas mayores, con énfasis en las personas y el trabajo con hábitos de vida saludables. Indican la necesidad de atención profesional para los trastornos depresivos en ancianos.

Transtornos Mentales; Salud Mental; Anciano

\section{Colaboradores}

F. S. A. Borim realizou a proposta do artigo, revisão da literatura, análise e interpretação dos dados, redação do texto. M. B. A. Barros e N. J. Botega colaboraram com a análise e interpretação dos dados, redação e revisão crítica do conteúdo intelectual.

\section{Agradecimentos}

À FAPESP pela bolsa de doutorado de F. S. A. Borim. Ao CNPq (Processo no 409747/2006-8) pelo financiamento da pesquisa e bolsa produtividade de M. B. A. Barros. À Secretaria Municipal de Saúde de Campinas pelo apoio financeiro. 


\section{Referências}

1. Veras R. Envelhecimento populacional contemporâneo: demandas, desafios e inovações. Rev Saúde Pública 2009; 43:548-54.

2. Instituto Brasileiro de Geografia e Estatística. Censo demográfico 2010 - características da população e dos domicílios. Resultados do universo. Rio de Janeiro: Instituto Brasileiro de Geografia e Estatística; 2010.

3. Clemente AS, Loyola Filho AI, Firmo JOA. Concepções sobre transtornos mentais e seu tratamento entre idosos atendidos em um serviço público de saúde mental. Cad Saúde Pública 2011; 27:555-64.

4. Blazer DG. Depression in late life: review and commentary. J Gerontol A Biol Sci Med Sci 2003; 58: 249-65.

5. Goldberg D, Huxley P. Common mental disorders: a biosocial model. London: Tavistock; 1992.

6. Fortes S, Villano LAB, Lopes CS. Nosological profile and prevalence of common mental disorders of patients seen at the Family Health Program (FHP) units in Petrópolis, Rio de Janeiro. Rev Bras Psiquiatr 2008; 30:32-7.

7. Maragno L, Goldbaum M, Gianini RJ, Novaes HMD, César CLG. Prevalência de transtornos mentais comuns em populações atendidas pelo Programa Saúde da Família (QUALIS) no Município de São Paulo, Brasil. Cad Saúde Pública 2006; 22:1639-48.

8. Ministério da Saúde. Portaria no 2528, de 19 de outubro de 2006. Aprova a Política Nacional de Saúde da Pessoa Idosa. Diário Oficial da União 2006; 19 out.

9. Tavares SMG. A saúde mental do idoso brasileiro e sua autonomia. Boletim do Instituto de Saúde 2009; 47:87-9.

10. Mari JJ, Willians P. A validity study of a psychiatric screening questionnaire (SRQ-20) in primary care in the city of São Paulo. Br J Psychiatry 1986; 148:23-6.

11. Scazufca M, Menezes PR, Vallada H, Araya R. Validity of the Self-Reporting Questionnaire-20 in epidemiological studies with older adults. Soc Psychiatry Psychiatr Epidemiol 2009; 44:247-54.

12. Gonçalves DM, Stein AT, Kapczinski F. Avaliação de desempenho do Self-Reporting Questionnaire como instrumento de rastreamento psiquiátrico: um estudo comparativo com Structured Clinical Interview for DMS-VI-TR. Cad Saúde Pública 2008; 24:380-90.

13. Costa JDS, Menezes ANB, Olinto MTA, Gicante DP, Macedo S, Brito MAP, et al. Prevalência de distúrbios psiquiátricos menores na cidade de Pelotas, RS. Rev Bras Epidemiol 2002; 5:164-73.

14. Marín-León L, Oliveira HB, Barros MBA, Dalgalarrondo P, Botega NJ. Social inequality and common mental disorders. Rev Bras Psiquiatr 2007; 29:205-3.

15. Rocha SV, Almeida MMG, Araújo TM, Virtuoso Jr. JS. Prevalência de transtornos mentais comuns entre residentes em áreas urbanas de Feira de Santana, Bahia. Rev Bras Epidemiol 2010; 13:630-40.

16. Lebrão ML, Laurenti R. Saúde, bem-estar e envelhecimento: o estudo SABE no Município de São Paulo. Rev Bras Epidemiol 2005; 8:127-41.
17. Maciel ACC, Guerra RO. Prevalência e fatores associados à sintomatologia depressiva em idosos residentes no Nordeste do Brasil. J Bras Psiquiatr 2006; 55:26-33.

18. Blay SL, Laks J, Nitrini R, Caramelli P. Epidemiologia dos transtornos mentais em idosos e a utilização dos serviços por esta população. In: Mello MF, Mello AAF, Kohn R, organizadores. Epidemiologia da saúde mental no Brasil. Porto Alegre: Editora Artmed; 2007. p. 143-50.

19. Corrêa AAM, Moreira-Almeida A, Menezes PR, Vallada $\mathrm{H}$, Scazufca $\mathrm{M}$. Investigating the role played by social support in the association between religiosity and mental health in low income older adults: results from the São Paulo Ageing \& Health Study (SPAH). Rev Bras Psiquiatr 2010; 33:157-64.

20. Dawson DA, Grant BF, Stinson FS, Zhou Y. Effectiveness of the derived Alcohol Use Disorders Identification Test (AUDIT-C) in screening for alcohol use disorders and risk drinking in the US general population. Alcohol Clin Exp Res 2005; 29:844-54.

21. Lima CT, Freire ACC, Silva APB, Teixeira RM, Farrel $\mathrm{M}$, Prince M. Concurrent and construct validity of the AUDIT in na urban Brazilian sample. Alcohol Alcohol 2005; 40:584-9.

22. Cervi A, Franceschini SC, Priore SE. Análise crítica do uso do índice de massa corporal para idosos. Rev Nutr 2005; 18:765-75.

23. Ludemir AB, Melo Filho DA. Condições de vida e estrutura ocupacional associadas a transtornos mentais comuns. Rev Saúde Pública 2002; 36:213-21.

24. Spijker J, Graaf R, Bijl RV, Beekman AT, Ormel J, Nolen WA. Duration of major depressive episodes in the general population: results from The Netherlands Mental Health Survey and Incidence Study (NEMESIS). Br J Psychiatry 2002; 181:208-13.

25. Lima MS, Soares BGO, Mari JJ. Saúde e doença mental em Pelotas, RS: dados de um estudo populacional. Rev Psiq Clín (São Paulo) 1999; 26:225-35.

26. Ludemir AB, Lewis G. Links between social class and common mental disorders in Northeast Brazil. Soc Psychiatry Psychiatr Epidemiol 2001; 36:101-7.

27. Rocha SV, Almeida MMG, Araújo TM, VirtuosoJunior JS. Atividade física no lazer e transtornos mentais comuns entre idosos residentes em um município do Nordeste do Brasil. J Bras Psiquiatr $2011 ; 60: 80-5$.

28. Rodrigues-Neto JF, Figueiredo MFS, Faria AAS, Fagundes M. Transtornos mentais comuns e uso de práticas de medicina complementar e alternativa - estudo de base populacional. J Bras Psiquiatr 2008; 57:233-9.

29. Gazalle FK, Lima MS, Tavares BF, Hallal PC. Sintomas depressivos e fatores associados em população idosa no Sul do Brasil. Rev Saúde Pública 2004; 38:365-71.

30. Wallerstein N. What is the evidence on effectiveness of empowerment to improve health? Copenhagen: World Health Organization Regional Office for Europe; 2006. 
31. Zaitune MPA, Barros MBA, César CLG, Carandina L, Goldbaum M. Fatores associados ao sedentarismo no lazer em idosos, Campinas, São Paulo, Brasil. Cad Saúde Pública 2007; 23:1329-38.

32. Culic V. Moderate drinking, psychological factors and cardiovascular protection. Eur Heart J 2009; 30:381-3.

33. Fillmore KM, Stockwell T, Chikritzhs T, Bostrom A Kerr W. Moderate alcohol use and reduced mortality risk: systemic error in prospective studies and new hypotheses. Ann Epidemiol 2007; 17(5 Suppl):16-23.

34. Skogen JC, Harvey SB, Henderson M, Stordal E, Mykletun A. Anxiety and depression among abstainers and low-level alcohol consumers. The Nord-Trondelag Health Study. Addiction 2009; 104:1519-29.

35. Zaitune MPA, Barros MBA, César CLG, Carandina L, Goldbaum M, Alves MCGP. Fatores associados à prática de atividade física global e de lazer em idosos: Inquérito de Saúde no Estado de São Paulo (ISA-SP), Brasil. Cad Saúde Pública 2010; 26 : 1606-18.

36. Lima MG, Barros MBA, César CLG, Carandina L, Goldbaum M, Alves MCGP. Comportamentos relacionados à saúde e qualidade de vida em idosos: um estudo de base populacional. Rev Saúde Pública $2011 ; 45: 485-93$.

37. Borim FSA, Barros MBA, Neri AL. Autoavaliação da saúde em idosos: pesquisa de base populacional no Município de Campinas, São Paulo, Brasil. Cad Saúde Pública 2012; 28:769-80.

38. Fillmore KM. Is alcohol really good for the heart? Addiction 2000; 95:173-4.

39. Rigo JC, Rigo JFO, Faria BC, Stein A, Santos VM. Trauma associado com uso de álcool em idosos. Brasília Med 2005; 42:35-40.

40. Pereira AR, Morita M, Barros MBA. Transtorno mental comum. In: Barros MBA, César CLG, Carandina $\mathrm{L}$, Goldbaum $\mathrm{M}$, organizadores. As dimensões da saúde: inquérito populacional em Campinas. São Paulo: Editora Hucitec; 2008. p. 143-52.
41. Costa E, Barreto SM, Uchoa E, Firmo JOA, Lima Costa MF, Prince M. Prevalence of International Classification of Diseases, 10th Revision common mental disorders in the elderly in a Brazilian community: The Bambui Health Ageing Study. Am J Geriatr Psychiatry 2007; 15:17-27.

42. Ratner PA, Johnson JL, Jeffery B. Examining emotional, physical, social and spiritual health as determinants of self-rated health status. Am J Health Promot 1998; 12:275-82.

43. Coelho FMC, Pinheiro RT, Horta BL, Magalhães PVS, Garcias CMM, Silva CV. Common mental disorders and chronic non-communicable diseases in adults: a population-based study. Cad Saúde Pública 2009; 25:59-67.

44. Gili M, Comas A, García-García M, Monzón S, Antoni SB, Roca M. Comorbidity between common mental disorders and chronic somatic diseases in primary care patients. Gen Hosp Psychiatry 2010; 32:240-5.

45. Alves LC, Leimann BCQ, Vasconcelos MEL, Carvalho MS, Vasconcelos AGG, Fonseca TCO, et al. A influência das doenças crônicas na capacidade funcional dos idosos do Município de São Paulo, Brasil. Cad Saúde Pública 2007; 23:1924-30.

46. McDougall FA, Kvaal K, Matthews FE, Paykel E, Jones PB, Dewey ME, et al. Prevalence of depres sion in older people in England and Wales: the MRC CFA Study. Psychol Med 2007; 37:1787-95.

47. Duarte MB, Rego MAV. Comorbidade entre depressão e doenças clínicas em um ambulatório de geriatria. Cad Saúde Pública 2007; 23:691-700.

48. Fleck MPA, Lima AFBS, Louzada S, Schestasky G, Henriques A, Borges VR, et al. Associação entre sintomas depressivos e funcionamento social em cuidados primários à saúde. Rev Saúde Pública 2002; 36:431-8

49. Hoffmann EJ, Ribeiro F, Farnese JM, Lima EWB. Sintomas depressivos e fatores associados entre idosos residentes em uma comunidade no norte de Minas Gerais, Brasil. J Bras Psiquiatr 2010; 59:190-7.

Recebido em 17/Set/2012

Versão final reapresentada em 14/Jan/2013

Aprovado em 25/Fev/2013 